Revue d'histoire de l'enfance « irrégulière »

Le Temps de l'histoire

16 | 2014

La part scolaire : jeunesse irrégulière et école $\left(\mathrm{XIX}^{\mathrm{e}}\right.$ $\mathrm{XX}$ siècles)

\title{
Une certaine conception de l'administration : Éducation nationale et enfance inadaptée (1945-1988), un témoignage
}

A certain idea of administrative work: French public educational services and maladjusted children (1945-1988), an account given by an inspector

\section{André Catteaux}

\section{(2) OpenEdition}

Journals

Édition électronique

URL : https://journals.openedition.org/rhei/3649

DOI : 10.4000/rhei.3649

ISBN : 978-2-7535-4019-4

ISSN : 1777-540X

Éditeur

Presses universitaires de Rennes

Édition imprimée

Date de publication : 30 octobre 2014

Pagination : 99-110

ISBN : 978-2-7535-3558-9

ISSN : 1287-2431

Référence électronique

André Catteaux, « Une certaine conception de l'administration : Éducation nationale et enfance

inadaptée (1945-1988), un témoignage », Revue d'histoire de l'enfance « irrégulière » [En ligne], 16 | 2014, mis en ligne le 30 octobre 2016, consulté le 08 septembre 2021. URL : http://journals.openedition.org/ rhei/3649; DOl : https://doi.org/10.4000/rhei.3649

Ce document a été généré automatiquement le 8 septembre 2021

(c) PUR 


\title{
Une certaine conception de l'administration : Éducation nationale et enfance inadaptée (1945-1988), un témoignage
}

\author{
A certain idea of administrative work: French public educational services and \\ maladjusted children (1945-1988), an account given by an inspector
}

André Catteaux

\section{NOTE DE L'ÉDITEUR}

Ce document est constitué d'extraits de deux témoignages rédigés par André Catteaux au début des années 2000, destinés initialement à sa famille et qu'il a confiés à Mathias Gardet pour libre publication. Le choix des extraits, les intertitres et les notes sont de la responsabilité de Laurent Besse qui remercie André Catteaux et Mathias Gardet pour leur générosité.

Le titre sous lequel est placé ce témoignage reprend celui d'un ouvrage où André Catteaux expose sa conception du rôle de l'inspecteur d'académie ${ }^{1}$. Acteur important de l'éducation spéciale de la seconde moitié du xxe siècle, il a exercé à partir de 1951 des fonctions d'inspecteur, qui l'ont conduit jusqu'à l'Inspection générale de l'Éducation nationale. Si l'enfance inadaptée n'a constitué qu'une partie des dossiers dont il avait la responsabilité, elle n'a cessé de le préoccuper, tant dans son action administrative que dans ce qu'il qualifie lui-même « d'activités militantes », à l'ANCE (Association nationale des communautés d'enfants devenue communautés éducatives) et surtout aux PEP (Pupilles de l'enseignement public), dont il est largement question ici. On saisit très bien à lire ce témoignage la place tenue après 1945 par le monde des œuvres liées à l'Éducation nationale dans la mise en place, par procuration, d'un quasi service public de l'enfance inadaptée : étroitement lié à l'administration, cet ensemble se nourrissait 
également du terreau militant enseignant laïque, celui des « associations amies de l'école ${ }^{2}$ ». La notion " d'œuvre complémentaire de l'enseignement public » prend tout son sens lorsqu'on lit le récit de la création de centres médico-psycho-pédagogiques (CMPP). La proximité entre administration et associations, dénoncée par certains, y était particulièrement forte et permettait d'agir vite et efficacement. Mais le fait de travailler entre membres de la grande famille Éducation nationale n'excluait pas les tensions, par exemple avec les représentants syndicaux, partenaires obligés de l'administration. Les divergences portent en particulier sur le degré d'intégration de ce quasi service public à l'institution Éducation nationale, certains syndicalistes réclamant l'assimilation pure et simple au système scolaire. André Catteaux prend position avec force contre une vision unificatrice qu'il qualifie de « sectaire ». Même si rien dans son témoignage ne permet de l'affirmer avec une absolue certitude, on peut supposer que les onze années passées à la tête de l'inspection académique du Morbihan, département français comptant le plus d'élèves scolarisés dans l'enseignement privé sous contrat, ne sont pas étrangères à sa conception des rapports entre l'école et ses partenaires.

Le mérite du témoignage d'André Catteaux est également de révéler ce qu'une action administrative et militante doit aux rencontres amicales mais également aux motivations personnelles ou familiales. L'administrateur ne cache pas que son expérience de père d'un enfant souffrant de dyslexie a compté dans son intérêt pour les CMPP. Mais on comprend également à le lire que le champ de l'enfance inadaptée fut pour lui un domaine d'autant plus stimulant qu'il s'agissait d'un secteur pionnier où beaucoup était à faire ou à inventer. Un tempérament bâtisseur trouvait là un terrain où s'exprimer, sans doute plus facilement que dans les autres domaines d'intervention d'un inspecteur, où les contraintes administratives, le poids des héritages rendaient le travail peut-être plus routinier et moins exaltant.

Laurent Besse

1 Le témoignage qui suit est essentiellement subjectif. J'évoque les relations entretenues au cours de ma carrière avec diverses associations complémentaires de l'enseignement public. Donc dans cette "pelote de mémoire ", je suis un fil directeur strictement personnel au gré des diverses fonctions que j'ai exercées : professeur d'École normale (1945-1951 à Arras), inspecteur primaire (1951-1967), inspecteur d'académie dans le Morbihan (1967-1978), inspecteur général (1978-1988). J'essaie de préciser les complémentarités, les occasions de collaboration, les interactions entre fonction publique et milieu associatif, les formes d'entraide, les avantages et les difficultés éventuelles. [...]

\section{Découverte de l'enfance inadaptée}

2 Mon premier contact avec l'enfance inadaptée date de 1947. Un certain nombre de centres avaient été créés en Forêt-Noire (alors zone d'occupation française) pour accueillir, pendant trois mois, des enfants issus de grandes villes françaises et qui souffraient encore de malnutrition ou de mauvaises conditions d'hygiène ou de logement. Pour assurer leur scolarité, $\mathrm{M}^{\mathrm{me}}$ Drouin ${ }^{3}$ fut chargée de recruter des enseignants. Comme le nombre des titulaires du CAEPA (certificat d'aptitude à l'enseignement des écoles de plein-air) était alors quasiment nul, elle eut l'idée d'assurer cette formation d'instituteurs spécialisés EPA (école de plein-air) dans un 


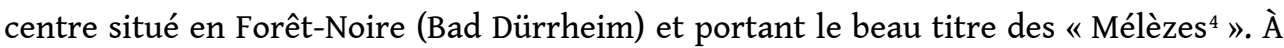
sa demande, je devins professeur préparant au CAEPA.

Devenu ultérieurement inspecteur primaire dans le Pas-de-Calais, j'avais dans ma circonscription une école de plein-air, maison d'enfants cas sociaux dite «Maison des Pupilles ». "Pupilles» est à prendre ici selon deux sens puisque les enfants étaient pupilles de l'Assistance publique et que la maison était gérée par l'OPEP, l'œuvre des pupilles de l'école publique ${ }^{5}$. À l'occasion d'une modification des circonscriptions, l'inspecteur d'académie du Pas-de-Calais amorça un début de spécialisation hors circonscription territoriale et, il me confia l'inspection des établissements d'hospitalisation et de cure situés essentiellement à Berck ${ }^{6}$. Je conservai la Maison des Pupilles. En outre l'inspecteur d'académie me chargea de le représenter dans les différentes instances départementales étudiant les problèmes des enfants et adolescents inadaptés (commission d'orientation des infirmes par exemple). Je pris quelques initiatives en organisant, en liaison avec les syndicats d'instituteurs et d'inspecteurs, des journées de présentation des problèmes de l'inadaptation. La manifestation la plus importante fut la réalisation d'une grande exposition de travaux d'élèves « L'École publique au service des malades » dans les locaux du casino municipal de Berck, en juin 1957. [...]

En outre, je continuais à m'intéresser aux problèmes des écoles de plein-air. Le Centre de Bad-Dürrheim fermé en 1949, la formation au CAEPA continua de 1951 à 1953 à l'École normale de filles d'Évreux, puis de 1955 à 1956, dans les locaux de l'école de plein-air de Suresnes, avant d'intégrer en octobre 1956 les bâtiments flambant neufs du CNEPA (Centre national des écoles de plein-air) de Suresnes. Durant toute cette période aucun professeur n'avait été nommé au Centre et des conférenciers assuraient l'enseignement. À ce titre, je fus souvent appelé à Évreux puis à Suresnes. C'est au cours de cette période que je fis connaissance avec l'ANCE (Association nationale des communautés d'enfants) association dans laquelle je m'engageais intensément tout au long de ma vie ${ }^{7}$. [...] Jacques Lacapère qui, avec son épouse Simone, dirigeaient une maison d'enfants bien connue "La Bastide de Beau Soucy» me sollicita pour une adhésion ${ }^{8}$. Cela ne se fit pas sans quelque hésitation! L'ANCE était alors surtout composée de directeurs de maisons d'enfants. Un inspecteur primaire pouvait-il être membre de l'ANCE? Et a fortiori membre du conseil d'administration comme je le devins très vite? Nulle hostilité à ma personne, mais problème de principe ! Résolu très vite et favorablement en définitive. Aujourd'hui, l'hésitation de ce temps parait bien futile. Toujours est-il que cela allait avoir un grand retentissement sur mes activités futures. [...]

\section{La création d'un CMPP avec les Pupilles de l'Oise}

Devenu IDEN, inspecteur départemental de l'Éducation nationale9 , dans l'Oise en 1958, sur un poste non spécialisé ${ }^{10}$, je continuais à m'intéresser à l'enfance inadaptée. En 1961, aidé par un collègue de l'École normale de Beauvais, je menais une grande enquête sur les enfants inadaptés dans le département de l'Oise et le ministère de l'Éducation nationale y accorda un certain intérêt. Je devins délégué départemental de l'ANCE; avec cette dernière association, j'organisais des journées d'information sur l'enfance inadaptée à l'intention du grand public. Pour les instituteurs de ma circonscription je prévoyais chaque année une visite dans un établissement spécialisé, 
soit pour les enfants infirmes moteurs, soit pour les enfants cas sociaux. Je continuais à suivre les activités de Suresnes quoique de façon moins intense. [...] Professionnellement, c'est avec les Pupilles que je travaillais en très étroite relation. En 1961, j'eus l'idée d'ouvrir un CMPP (centre médico-psycho-pédagogique) à Beauvais. [...]

Ici se mêlent étroitement les soucis personnels et les engagements professionnels. [ $\mathrm{L} a$ dyslexie de son fils avait conduit André Catteaux à découvrir le centre d'aide psychopédagogique annexé à l'École normale d'Auteuil à Paris] Même si [notre fils] n'a jamais fréquenté le CMPP de Beauvais, c'est quand même lui qui en fut, psychologiquement et affectivement l'inspirateur. Je n'ai jamais regretté cette création ! [...]

Comme il n'était pas possible de créer un tel établissement sous statut Éducation nationale, je décidais d'y intéresser les Pupilles, afin de rester le plus proche du service public. [...] Le premier à convaincre c'était M. Blanchard, à la fois comme inspecteur d'académie et comme président de l'association des PEP (Pupilles de l'enseignement public). Comme inspecteur d'académie, il accepta de mettre des enseignants à la disposition du CMPP. Comme président des Pupilles, il décida le conseil d'administration à prendre en responsabilité la gestion du centre. Ce fut assez facile. M. Blanchard me soutenait en tout, le conseil d'administration des Pupilles comptait beaucoup d'enseignants militant déjà dans d'autres œuvres (MGEN, MAIF ${ }^{11}$ ); en outre, je savais que la gestion du centre ne coûterait rien aux Pupilles, bien au contraire, c'était l'heureuse époque où tout était facile. [...]

Ensuite, le maire de Beauvais, un excellent homme, d'une très grande ouverture d'esprit, nous aménagea des locaux préfabriqués pour abriter le CMPP. Mais le souvenir qui me reste le plus prégnant, c'est l'enthousiasme dont fit preuve le directeur de la caisse de Sécurité sociale de Beauvais. Il me soutint bien au-delà de ce que sa seule fonction exigeait. [...] Les CMPP étaient alors financés par un " prix de séance » fixé par le préfet et payé par la Sécurité sociale. Celle-ci, en ces heureux temps, n'était soumise à presque aucune limitation financière comme c'est le cas aujourd'hui. Une fois fixé, le prix de séance était reconduit chaque année avec les augmentations annuelles justifiées. Aussi, au départ, la prudence et l'astuce, dirais-je même, consistaient à obtenir le prix de séance le plus élevé possible. J'obtins que le prix de séance du CMPP de Beauvais soit aligné sur les prix des autres CMPP existants. C'était un gros avantage car le CMPP de Beauvais comptait beaucoup de personnel mis à disposition par l'Éducation nationale, ce qui n'entamait pas le budget propre du centre. Si bien qu'à ses débuts, le CMPP de Beauvais fit des bénéfices considérables. [...]

\section{Réticences syndicales et universitaires}

Certes il y eut des difficultés. Sur le plan départemental, je me heurtais à l'indifférence plus qu'à une réelle résistance du Syndicat des instituteurs ${ }^{12}$. Ses membres n'étaient pas contre la création d'un CMPP, mais pour eux, laïcité, service public et Éducation nationale, tout se confondait. Ils voulaient bien d'un CMPP, mais à condition que celuici soit non seulement public, mais aussi directement géré par l'Éducation nationale, comme une école ordinaire. [...] Je retrouverai plus tard en 1981 cette vision hallucinée et sectaire : tout service doit être public, et, en outre, dès que dans un service public, on introduit la moindre goutte de pédagogie ou de psychologie, le service doit être non seulement public mais aussi géré par l'Éducation nationale ${ }^{13}$. À la limite pour certains laïques farouches, la moitié de la France serait Éducation nationale. Fort heureusement, 
je trouvais auprès d'autres instances, beaucoup plus de compréhension et même de la passion. [...] Une difficulté plus inattendue se présenta au niveau national lors des contacts préparatoires à la création du CMPP. En 1961-1962 n'existaient encore en France que 6 ou 7 CMPP dont l'ancêtre Claude Bernard ${ }^{14}$. Ils s'étaient regroupés au sein d'une Association française des CMPP qui jouait un rôle plus important sur le plan moral et psychologique que sur le plan administratif. Cette association mettait en valeur certains principes et n'accordait son soutien, en fait un label de qualité, qu'à certaines conditions. Elle préconisait pour tout centre une double direction : direction médicale assurée par un médecin psychiatre qualifié en psychanalyse, direction administrative et pédagogique assurée dans les centres existants jusqu'alors par des professeurs de faculté. Je tenais en démarrant Beauvais à obtenir l'appui de l'Association française. J'obtins un rendez-vous avec la présidente de l'association, une universitaire de grande réputation, $\mathrm{M}^{\mathrm{me}}$ Favez-Boutonnier ${ }^{15}$. Elle se réjouit de la création d'un nouveau CMPP, mais pour lui donner sa caution et accepter son adhésion à l'association, elle souleva un problème auquel je ne m'attendais pas. J'avais pourtant prévu la double direction. Comme directeur médical, j'avais sollicité un médecin du service public, chef de service à l'hôpital psychiatrique de Clermont de l'Oise. Bien qu'il ne fût pas psychanalyste, Mme Favez-Boutonnier déclara s'en contenter. Il n'en alla pas de même pour la direction pédagogique. J'avais prévu qu'elle serait assurée par un instituteur rééducateur en psychopédagogie, ayant fait le stage de spécialisation à Beaumont. (C'est d'ailleurs le cas aujourd'hui dans la plupart des CMPP). Mais en 1961, $\mathrm{M}^{\mathrm{me}}$ Favez-Boutonnier fit une objection majeure ; elle ne concevait de CMPP que dirigé par un universitaire. L'esprit de corps n'est pas un vain mot dans l'Université française ! $\mathrm{M}^{\mathrm{me}}$ Favez-Boutonnier ne finit par accepter de donner sa «bénédiction » au CMPP de Beauvais qu'à l'expresse condition que j'en assure personnellement la direction! Curieux compromis, avouons-le! Malgré la charge de travail supplémentaire que cela constituait, j'acceptais. Ainsi fut, pour ainsi dire, «adoubé » le premier CMPP des Pupilles. Je rédigeai alors un petit document sur « les conditions d'agrément et de fonctionnement d'un CMPP », largement diffusé au début par les PEP qui comptent maintenant dans leurs rangs, bien plus d'une centaine de CMPP.

\section{Fidélité à l'ANCE}

10 Naturellement un de mes premiers soucis fut de faire adhérer à l'ANCE le CMPP de Beauvais, puis celui de Compiègne que j'avais créé dans la foulée. Bien entendu, le même problème se posa: un CMPP était-il une communauté d'enfants? Je fis remarquer qu'à tout le moins, c'était une communauté d'adultes et l'affaire fut réglée. Avec un collègue nous lançâmes au sein de l'ANCE une Commission des CMPP ; très active, elle compta plusieurs centaines de CMPP et se continue aujourd'hui sous un autre nom malgré la disparition de l'ANCE.

11 Je reconnais que l'ANCE fut l'association dans laquelle je m'engageais le plus assidûment. Très vite nommé vice-président, j'y restais une trentaine d'années [alors qu'André Catteaux était devenu inspecteur d'académie puis inspecteur général]. Et surtout pendant une quarantaine d'années, j'ai animé la Commission psychopédagogique, créée dès 1954, chargée entre autres de préparer le congrès annuel de l'ANCE : choix du thème et des intervenants, organisation de groupes de travail préparation de la synthèse. 


\section{Inspecteur d'académie dans le Morbihan et militant associatif}

13 [En 1967, André Catteaux devient inspecteur d'académie du Morbihan, à Vannes. Il dirige donc l'ensemble des services de l'Éducation nationale de ce département, sous l'autorité du recteur de l'académie de Rennes.]

14 Le métier d'inspecteur d'académie (IA), peut être envisagé sous son aspect officiel, à savoir les strictes obligations liées à la seule fonction, chacun pouvant d'ailleurs donner au métier une coloration différente et s'y engager avec plus ou moins de conviction. Mais cette conviction peut également s'étendre au-delà des seules exigences professionnelles, tout en restant liée à la vocation éducative, à savoir rester au service des enfants et de l'école. Ainsi existent au sein de la sphère éducative une multiplicité d'œuvres et d'associations auxquelles l'IA a toute liberté d'apporter ou non son soutien. Je puis affirmer, et tous l'ont reconnu, que durant mon séjour à Vannes, j'ai beaucoup contribué à soutenir et même à développer les œuvres post et périscolaires. J'agissais alors au sein du département, en militant associatif plus qu'en simple fonctionnaire. [...]

15 J'accorde ici la priorité à l'action que j'ai menée en faveur des PEP du Morbihan et cela pour deux raisons, l'une de forme, l'autre de fond. La raison de forme compte peu ; elle a d'ailleurs actuellement tendance à s'estomper. Mais en principe, c'est l'association la plus étroitement liée à l'Éducation nationale: les statuts de chaque association départementale des Pupilles prévoient en effet, que l'inspecteur d'académie en est de droit le président. À mon arrivée à Vannes, j'acceptai bien évidemment cette fonction et à l'époque tous les IA faisaient de même. [...] Mais si dans ce récit, j'accorde la priorité aux Pupilles, ce n'est pas pour cette raison formelle de présidence ; c'est que les Pupilles fut l'œuvre en faveur de laquelle mon action a été la plus spectaculaire.

16 J'étais à peine arrivé à Vannes que $\mathrm{M}^{\text {lle }}$ Escoubet, l'inspectrice des écoles maternelles, devenue depuis une excellente amie, m'annonçait en souriant : « Nous vous attendions, Monsieur l'inspecteur d'académie pour créer un CMPP ! " Ma réputation de Beauvais m'avait précédé. Un an après mon arrivée, le CMPP de Vannes était ouvert. Mon action a surtout consisté à aider Mlle Escoubet. Elle a joué dans le Morbihan, le rôle qui fut le mien dans l'Oise. [...]

17 J'obtins également que mon épouse puisse exercer à plein temps au CMPP, comme rééducatrice en psychomotricité, tout en étant payée par l'Éducation nationale comme agent de bureau ; en somme un poste administratif « Éducation nationale » était affecté au CMPP. C'était un avantage énorme qui, je dois l'avouer franchement, était dû à ma situation d'inspecteur d'académie. À Beauvais, ma femme était dans une situation administrative régulière: elle était payée sur un poste d'agent de bureau créé à l'inspection académique pour exercer le secrétariat de l'inspection primaire. Elle travaillait au CMPP de Beauvais, à titre privé en heures supplémentaires. À Vannes, en 1967, aucun poste n'était disponible à l'inspection académique, sur lequel mon épouse aurait pu être nommée. En outre, je ne tenais pas à ce que la femme de l'inspecteur d'académie travaillât dans les services de l'inspection académique, ce qui aurait pu créer des situations délicates. Je m'en expliquai avec le recteur qui, en 1968, créa pour 
ma femme un poste d'agent de bureau à l'inspection académique de Vannes, avec exercice au CMPP ; je lui en fus reconnaissant. Et pour que sa situation soit maintenant parfaitement claire sur le plan administratif, elle prit sa retraite anticipée de l'Éducation nationale (retraite qu'elle ne touchera qu'à 60 ans) et elle continuera à travailler au CMPP de Vannes, à titre privé comme employée de l'œuvre des Pupilles, gestionnaire du CMPP.

Pour en revenir donc au CMPP de Vannes, il devint très vite florissant et les locaux aménagés provisoirement dans une école devinrent insuffisants. Et c'est une de mes fiertés d'avoir réalisé la construction de locaux neufs. [...] Si bien que, sur la lancée, après avoir créé le CMPP de Vannes en 1968, les Pupilles du Morbihan créèrent celui de Lorient en 1969, avec construction neuve en 1975, et celui de Pontivy en 1971. Ce rayonnement des CMPP n'a fait que croitre après mon départ. C'est ma meilleure récompense. [...]

\section{Un conte de fées administratif et pédagogique}

19 Je pourrais en être fier, mais les choses me paraissent aujourd'hui si lointaines! Nous sommes en juillet 1976. Une association de professeurs de l'enseignement technique spécialistes des métiers du bois - d'où le nom de l'association " Amibois » - décide de tenir son congrès annuel à Vannes. J'y fus invité [...] Ce congrès Amibois 1976 marque le début d'une aventure, un quasi conte de fées, conte de fées administratif et pédagogique, bien entendu. Je fis la connaissance au cours de ce congrès du président d'Amibois ; c'était un professeur de l'École normale nationale d'apprentissage (ENNA) ${ }^{16}$ à Saint-Denis, M. Eulacia ${ }^{17}$, un homme sympathique et d'un dynamisme assez étonnant. Il exprimait comme principe que ce qui soude le mieux les hommes, c'est moins ce qu'ils disent que ce qu'ils font ensemble, surtout quand il s'agit de professionnels. Ainsi voulait-il proposer aux membres de son association une grande œuvre à mener en commun. Cette idée exposée au hasard d'une conversation autour d'un pot, fit " tilt » dans mon esprit. "Et si je vous proposais un projet?" Ainsi naquit un jour de juillet 1976, ce que plus tard, nous avons appelé « l'opération Arzal»!

Le projet réunissait deux associations, Amibois et les Pupilles du Morbihan, en vue de la réalisation d'un centre permanent de classes de mer pouvant également être utilisé comme colonie de vacances. Le plus original évidemment était la participation d'Amibois avec un intérêt pédagogique incontestable. Le centre devait comporter un certain nombre de chalets destinés à loger chacun une douzaine d'enfants. Les dossiers techniques et pédagogiques devaient être réalisés par les stagiaires de l'ENNA, futurs professeurs de l'enseignement technique. Suivant ces plans, la réalisation matérielle, c'est-à-dire la construction et l'aménagement des chalets devait être effectuée par des collèges d'enseignement technique volontaires, répartis sur l'ensemble de la France. Les Pupilles devaient d'abord fournir le terrain, ce qui ne fut possible que grâce à l'aide généreuse du conseil général et du préfet Baudequin avec qui j'entretenais des relations amicales. Le département offrit gracieusement un terrain situé près du barrage d'Arzal, à l'embouchure de la Vilaine. Les Pupilles s'engageaient en outre à construire un bâtiment pour abriter les services généraux. Un architecte ami, qui avait déjà construit le CMPP de Lorient, nous assurerait une participation quasi bénévole. Néanmoins l'investissement financier restait de taille; mais là encore l'aide du préfet s'avéra 
précieuse. Il nous réserva la presque totalité des crédits de la Jeunesse et des Sports destinés aux constructions de colonies de vacances. [...]

\section{Perpétuer les partenariats, par-delà les individus}

Mais qu'advint-il en fait de ce projet? Les choses commencèrent très bien : un chalet expérimental fut construit dans un CET (collège d'enseignement technique), et fut exposé lors du congrès 1978 de l'association Amibois qui avait lieu cette année-là à Murat en Auvergne. J'y fus invité. Je trouvai le chalet magnifique et je me réjouis de ces prémices encourageantes. Mais en octobre 1978, je quittais le Morbihan. Peu de temps après le préfet Baudequin également: il fut nommé préfet de région à Poitiers. Ses promesses pour un financement quasi total de la construction revenant aux Pupilles ne furent pas tenues par son successeur. Les Pupilles devaient donc faire appel à l'emprunt et mon propre successeur s'est montré hésitant, prêt à abandonner le projet. Finalement, grâce à l'intérêt manifesté par les autorités ministérielles de l'Éducation nationale, grâce également à certains membres du conseil d'administration des Pupilles, l'opération finit par se réaliser, avec quelque retard, durant les vacances 1981 ou 1982. [...]

La manière dont l'œuvre des PEP du Morbihan évolua après mon départ me laisse quelque amertume. Mes divers successeurs s'y intéressèrent de moins en moins. Certes, un inspecteur d'académie ne peut pas personnellement participer à toutes les activités de toutes les associations. J'avais trouvé une méthode pour assurer dans les œuvres, un engagement compatible avec le temps dont je disposais. Dans chaque œuvre, je m'étais arrangé pour qu'un IDEN s'intéressât aux activités concernées par cette œuvre. C'est lui qui me représentait aux réunions de bureau ou de conseil d'administration. En ce qui concerne les Pupilles, j'avais réussi à faire élire comme vice-président, Gilbert Hautot, un inspecteur distingué, dévoué, cultivé, à tel point qu'il fut nommé inspecteur d'académie en 1980. [...]

[Par la suite, au cours des années 1980] l'opposition syndicat-administration devint si vive qu'elle en retomba sur l'œuvre des Pupilles. L'IA restait président des Pupilles, mais ne s'y intéressait nullement ; il critiquait même l'action des Pupilles en fait animée par le vice-président délégué syndical ${ }^{18}$. Un jour, m'a-t-on conté, l'IA envoya une lettre de remontrances au président des Pupilles!... Il s'écrivait ainsi à lui-même pour s'admonester...cela fit sourire! L'un des derniers IA arrivés, pour ramener, dit-il, le calme, et éviter les hostilités avec le syndicat finit par abandonner la présidence des Pupilles, la laissant officiellement au délégué syndical. J'avoue que a posteriori cela me fait mal, moins sur le plan personnel que pour des raisons de principe : je regrette vivement que des IA se désintéressent d'œuvres crées pour le bien des enfants. Et il paraît que bien d'autres départements suivent le mauvais exemple du Morbihan : l'IA y abandonne la présidence des Pupilles; c'est dommage !19

[...]

\section{En guise de bilan}

$\mathrm{Au}$ terme de ce témoignage purement personnel et factuel et en guise de bilan, j'aimerais en quelques lignes dégager, tels que je les ai ressentis, la nature des rapports 
entre un fonctionnaire de l'Éducation nationale et les associations, ainsi que le rôle que je crois avoir joué dans chacune de ces fonctions. Comme professeur (d'École normale), le bilan est vite fait: une quasi-ignorance réciproque. En tant qu'IDEN, ce sont les rapports d'amitié et de confiance tant avec mes instituteurs qu'avec les responsables d'associations qui me semblent l'emporter et mon rôle s'est surtout borné à de l'incitation (sauf à Beauvais pour la création du CMPP). C'est comme inspecteur d'académie par contre que j'ai vivement ressenti le poids des responsabilités et mon rôle fut surtout celui de création et de développement dans le domaine associatif. Enfin, l'Inspection générale m'a laissé, je crois, une bien plus grande liberté de choix et j'ai le sentiment d'y avoir surtout joué, plus qu'ailleurs, un rôle pédagogique d'animation et de formation.

\section{NOTES}

3. Marcelle Drouin (1892-1983), inspectrice générale des écoles maternelles. Aprèsguerre, elle avait en charge la question des enfants déficients physiques. Elle est à l'origine de la création du Centre national des écoles de plein-air de Suresnes en 1954.

4. Sur ce sujet, voir CATTEAUX André, «Au berceau du Centre de formation des maîtres des Écoles de Plein-Air (1945-1949)» dans CHÂTELET Anne-Marie, LERCH Dominique, LUC Jean-Noël, L'École de plein-air : une expérience pédagogique et architecturale dans l'Europe du $\mathrm{XX}$ siècle. Open-air schools: an educational and architectural venture in twentieth-century Europe, Paris, Recherches, 2003, p. 296-304.

5. L'CEuvre des pupilles de l'école publique, ou Pupilles dans le texte, aujourd'hui connue sous le nom des PEP, a été fondée en 1915 pour venir au secours des orphelins de guerre. Étroitement liée à l'Éducation nationale, tout en conservant un statut associatif, elle s'est ensuite tournée vers l'action sanitaire puis médico-sociale. Sur son histoire: GARDET Mathias, Histoire des PEP, Pupilles de l'école publique, Paris, Beauchesne, 2008, vol. 1 «1915-1939: La solidarité, une solidarité laïque». Le second volume consacré à la période de 1940 à nos jours doit paraître chez le même éditeur fin 2014.

6. Berck, commune balnéaire du Pas-de-Calais, est connue depuis le Second Empire, pour ses nombreux établissements hospitaliers et médicaux destinés en particulier aux enfants.

7. L'ANCE (Association nationale des communautés d'enfants devenue communautés éducatives) a été fondée en 1949 et a été dissoute en 2003. Sur son histoire : GARDET Mathias, «L'Association nationale des communautés d'enfants et les écoles de plein-air. D'un idéal de vie à un idéal laïque (1949-1959)», incHÂTELET Anne-Marie, LERCH Dominique, LUC Jean-Noël, L'École de plein air..., op. cit., p. 247-254.

8. Jacques Lacapère (1914-1997) et Simone (1916-2007), tous deux instituteurs, sont des figures majeures de l'éducation spéciale. Voir leurs notices biographiques dans le Dictionnaire biographique Maitron : [http://maitron-en-ligne.univ-paris1.fr/]. 
9. IDEN était le nom des inspecteurs du premier degré, en charge d'une circonscription d'enseignement primaire, aujourd'hui IENCCPD, inspecteurs de l'Éducation nationale chargés d'une circonscription du premier degré.

10. Les postes d'inspecteur spécialisé en charge de l'enfance inadaptée (et non pas d'une circonscription), sont créés par la circulaire du 10 octobre 1961. Mais le poste occupé par André Catteaux n'a été requalifié qu'à son départ en 1967.

11. MGEN : Mutuelle générale de l'Éducation nationale, MAIF : Mutuelle assurance des instituteurs de France. Il est intéressant de noter qu'André Catteaux, utilise le terme "œuvre» qui signifie juridiquement «œuvre complémentaire de l'enseignement public » pour désigner les PEP (qui sont au sens strict une œuvre) et la MAIF et la MGEN qui appartiennent à la galaxie enseignante mais n'en sont pas.

12. Le SNI (Syndicat national des instituteurs) qui, en raison du paritarisme, est un partenaire essentiel de l'administration, surtout au niveau départemental. Par ailleurs, il se fait le défenseur de la laïcité, qui dans les années 1950-1970, se confond avec la vision d'une Éducation nationale conquérante. Voir AUBERT Véronique, BERGOUGNIOUX Alain, MARTIN Jean-Paul, MOURIAUX René, La forteresse enseignante: La Fédération de l'Éducation nationale, Paris, Fayard, 1985.

13. Allusion aux projets de création d'un grand service public laïque et unifié de l'Éducation nationale (SPULEN) que la FEN (Fédération de l'Éducation nationale) espérait voir mettre en œuvre par François Mitterrand à partir de 1981. S'il s'agissait pour la gauche enseignante de régler définitivement la question de l'enseignement privé, cette vision intégratrice s'accompagnait, chez certains militants, d'une volonté d'englober dans un vaste ministère de l'Éducation nationale l'ensemble des services concernant l'éducation, en « nationalisant » des initiatives privées ou associatives.

14. Créé en 1946 dans les locaux du lycée Claude Bernard dans le $16^{\mathrm{e}}$ arrondissement parisien.

15. Juliette Favez-Boutonnier (1903-1994), professeur à la Sorbonne, fondatrice de la Société française de psychanalyse avec Daniel Lagache.

16. Les ENNA formaient les professeurs des lycées professionnels avant1991.

17. Henry Eulacia (1926-2011), enseignant-formateur en menuiserie puis directeur d'ENNA, fondateur d'Amibois, association amicale des professeurs de l'enseignement technique des métiers du bois [http://www.amibois.com/].

18. C'est-à-dire responsable départemental du SNI.

19. Il s'agit d'une tendance générale, l'administration de l'Éducation nationale souhaitant mieux distinguer ce qui relève des prérogatives administratives «publiques » de ce qui serait du domaine d'une initiative associative «privée ».

1. CATTEAUX André, L'Inspection académique : une certaine conception de l'administration départementale dans l'éducation, Rennes, CRDP, 1976.

2. Sur les associations amies de l'école : GEROME Noëlle, « Une étude organisationnelle en sociologie de l'éducation : l'enseignement primaire dans un département français », Revue française de sociologie, 8 (1967), p. 98-116. 


\section{RÉSUMÉS}

André Catteaux témoigne au sujet de son action en faveur de l'enfance inadaptée, en sa double qualité de fonctionnaire et de militant associatif, deux dimensions étroitement liées chez lui. Professeur d'École normale, chargé également de former les instituteurs spécialisés, il devint inspecteur primaire dans le Pas-de-Calais puis l'Oise (1951-1967) avant de devenir inspecteur d'académie du Morbihan en 1967 puis inspecteur général en 1978. Il œuvra en particulier à la création de centres médico-psycho-pédagogiques (CMPP). Dans son témoignage, il met en valeur l'imbrication entre l'administration et les associations complémentaires de l'école comme les Pupilles de l'enseignement public (PEP) qui permettent à l'administration de créer des quasi services publics tout en bénéficiant de la souplesse de fonctionnement du monde associatif. L'engagement d'André Catteaux en faveur de l'enfance inadaptée l'a conduit également à exercer des responsabilités au sein de l'ANCE (Association nationale des communautés d'enfants).

André Catteaux gives an account of his action in favor of maladjusted childhood as a civil servant and as a community activist, two interwoven dimensions of his career. Teacher in a School of Education (Ecole normale), also in charge of training masters specialized in dealing with backward children, he became a primary school inspector in Pas-de-Calais then in Oise (1951-1967) before becoming Head of Education services for the department of Morbihan in 1967, then National Chief inspector in 1978. He acted in favor the opening of Centres medico-psychopédagogique (CMPP), counselling centres devoted to children with severe school maladjustment. In his account, he draws particular attention to the close connections between Education services and pro-school associations like Pupilles de l'enseignement public which enables the ministry of Education to create quasi public services while benefiting from the flexibility coming from the way the associative world functions. His commitment towards maladjusted childhood resulted in his taking responsibilities in ANCE (Association nationale des communautés d'enfants).

\section{INDEX}

Mots-clés : enfance inadaptée, inspecteur de l'Éducation nationale, CMPP (centre médicopsycho-pédagogique), association, 1945 à nos jours

Keywords : maladjusted childhood, French education inspector, CMPP, charity and association, from 1945 onwards

\section{AUTEUR}

\section{ANDRÉ CATTEAUX}

André Catteaux est inspecteur général honoraire de l'Éducation nationale. Il a œuvré en particulier dans le domaine de l'enfance inadaptée. 